\title{
Clinicopathologic Characteristics of Gastroenteropancreatic Neuroendocrine Tumors: Experience of a National Referral Hospital in Indonesia
}

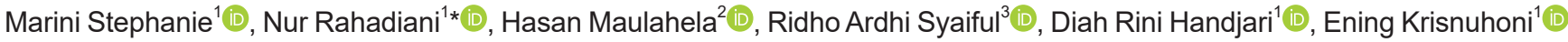 \\ ${ }^{1}$ Department of Anatomical Pathology, Division of Gastrointestinal and Hepatobilliary Pathology, Faculty of Medicine, Universitas \\ Indonesia, Dr. Cipto Mangunkusumo National Referral Hospital, Jakarta, Indonesia; ${ }^{2}$ Department of Internal Medicine, Division \\ of Gastroenterology, Faculty of Medicine, Universitas Indonesia, Dr. Cipto Mangunkusumo National Referral Hospital, Jakarta, \\ Indonesia; ${ }^{3}$ Department of Surgery, Division of Digestive Surgery, Faculty of Medicine, Universitas Indonesia, Dr. Cipto \\ Mangunkusumo National Referral Hospital, Jakarta, Indonesia
}

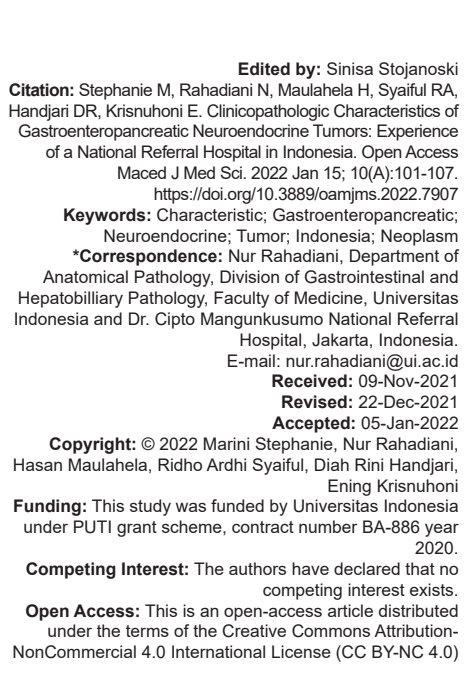

\section{Introduction}

Gastroenteropancreatic

neuroendocrine tumors (GEP-NETs), which can arise from any site in the gastrointestinal tract, share the expression of neuroendocrine markers, such as chromogranin and synaptophysin. The incidence of GEP-NET has steadily increased over the past several years, but the incidence depends on the primary site. Esophageal NETs are very rare and account for approximately only $0.04-1 \%$ of all esophageal cancers. Moreover, the incidence of GEPNETs in the gastrointestinal tract is highest in the small intestine $(1.25 \%)$, followed by the rectum $(1.18 \%)$, pancreas $(0.85 \%)$, stomach $(0.45 \%)$, and appendix $(0.38 \%)$, according to the surveillance, epidemiology, and end results (SEER) program database [1], [2].

\begin{abstract}
BACKGROUND: Gastroenteropancreatic neuroendocrine tumors (GEP-NETs) have variable biological behavior although they are all malignant.

AIM: This study aimed to present the 10-year prevalence along with the clinicopathologic characteristics of GEPist of patients with possible GEP-NET diagnosis using ICD-O code for topography and morphology. Cases were metastasis, hormone status, and chief complaints. Pathological characteristics included the type of GEP-NET, RESULTS: A total of 84 cases of GEP-NET from 2009 to 2019 were included in the study; of these, $38.1 \%$ were NETs, $28.6 \%$ were neuroendocrine carcinomas, and $33.3 \%$ were mixed neuroendocrine-non-neuroendocrine originated from the rectum $(21.4 \%)$ and were mostly non-functioning $(90.5 \%)$ with an average tumor size of $4.77 \mathrm{~cm}$. Most tumors were localized (53.6\%), but metastasis was found in $28.6 \%$ of cases. LVI was positive in $35.7 \%$ of with unknown primary sites, dissemination, LVI, and larger tumor size.
\end{abstract}

CONCLUSION: GEP-NETs can arise from any site in the gastrointestinal tract and have variable clinicopathologic characteristics. Primary site, stage, LVI, and tumor size are associated with grade.
A retrospective study in China reported the majority of mortality among GEPNETs patients' were related to the tumor, reached at $57.9 \%$ ( $n=22$ out of 36 ). Age and metastases on presentation were significant predictors of mortality and disease progression, while the higher tumor grade also independently predicted disease progression [3]

GEP-NETs are all malignant and have variable biological behavior, depending on tumor size, histological differentiation, and Ki67 index [4]. GEPNET classification has changed over time. In early classification schemes, pathologists classified GEPNET locations into the foregut (stomach, duodenum, upper jejunum, and pancreas), midgut (lower jejunum, ileum, appendix, and cecum), and hindgut (colon and rectum). The classification changed into well-differentiated, poorly differentiated, and mixed 
neuroendocrine-non-neuroendocrine (MiNEN) tumors throughout the following decade, irrespective of the site of origin. This classification generally differentiates the biological behavior into indolent and high-grade aggressive carcinoma, which has different therapeutic approaches. Surgery is the treatment of choice for low-grade tumors, while chemotherapeutic agents for unresectable and high-grade tumors. Low-grade tumors can resemble high-grade aggressive carcinomas histologically, demonstrating their unpredictable behavior regardless of their histological features and grade [5], [6].

Indonesia has limited centers that can perform a diagnostic panel for GEP-NETs, and thus, this disease entity has not yet attracted national attention. Studies of GEP-NETs have not been extensively performed or published, and to date, no previous study has reported GEP-NETs in Indonesia. This study presents the 10-year prevalence along with the clinicopathologic characteristics of GEP-NETs and their association with tumor grade at a national referral hospital in Indonesia. Knowledge of the clinical and pathological profiles and their association with tumor grade will be needed to expand further research in this field.

\section{Patients and Methods}

This is a cross-sectional study of patients with GEP-NETs who presented to our hospital, a national referral hospital in Indonesia, from 2009 to 2019. Data were obtained retrospectively from patient archives in the department of anatomical pathology and hospital medical records. We performed a total sampling of all available subjects by extracting list of patients with possible GEP-NET diagnosis using ICD-O code for topography and morphology and found 106 patients. The studies have been approved by the ethics committee with protocol number 20-05-0557 and have been performed in accordance with the ethical standards as laid down in the 1964 Declaration of Helsinki and its later amendments.

$\begin{array}{llr}\text { Two } & \text { independent pathologists } \\ \text { blindly confirmed the diagnosis by examining }\end{array}$ each patient's hematoxylin- and eosin-stained slides for histomorphology and assessing the immunohistochemical findings. Agreement between two pathologists is almost perfect indicated by kappa value 0.90 . When a disagreement arose between the two independent pathologists, a third pathologist examined the slides. Neuroendocrine markers used for immunohistochemistry were chromogranin $A$, synaptophysin, and CD56. Ki67 was used to classify a tumor grading. Carcinoembryonic antigen staining was performed in cases suspected to be MiNEN. Cases were included in the study if histomorphological findings were indicative of GEP-NET and if the diagnosis was confirmed by immunohistochemistry staining (positive for chromogranin A or positive staining in at least two of three neuroendocrine markers being used). To minimize the risk of bias, data on the clinical characteristics were obtained from hospital medical records by an independent observer who was not informed about the patient's diagnosis. Patients with incomplete data from the hospital medical records or with missing tissue slides were excluded from the study. This process excluded 22 patients, yielding 84 patients who were included. In the grading analysis, three patients were excluded since we were unable to stain the slides for Ki67 because the blocks belonged to outside referring hospital.

The clinical characteristics included age, sex, primary sites, tumor stage, metastasis, hormone status, and chief complaints. Treatment and radiologic modalities were also recorded. The tumor stage was defined as the extension of the tumor and was classified as localized, locoregional, and distant metastasis. The locoregional extension applies to tumors that invade surrounding lymph nodes or organs. Tumor stage and metastasis data were obtained from pathology resection reports or by radiologic modalities. In terms of hormone status, tumors were classified as functioning or non-functioning according to symptoms. Pathological characteristics included type of GEP-NET, specimen type, grade, and lymphovascular invasion (LVI). The types of GEP-NET are neuroendocrine tumor (NET), neuroendocrine carcinoma (NEC), and MiNEN. NET was classified as $\mathrm{G} 1, \mathrm{G} 2$, or $\mathrm{G} 3$, which correspond to a Ki67 index of $<3,3-20$, and $\geq 20$, respectively. NEC was classified as a small cell (SCNEC) and large cell (LCNEC) NEC, depending on tumor morphology, as assessed by pathologists. The grade was determined to be a high (NET G3, NEC, and MiNEN) or low grade (NET G1, G2). LVI was defined as positive or negative. Some specimens were undefined and listed as "not available" in the biopsy report.

Data were then processed and analyzed using SPSS version 20 for the univariate analysis; a $\chi^{2}$ test and t-test were used to compare categorical and numerical variables between tumor groups, respectively. A post hoc analysis was performed for variables other than those with a $2 \times 2$ design. Frequency, proportion, mean, and standard deviation are presented accordingly.

\section{Results}

\section{Clinical characteristics}

Of the 84 patients with GEP-NETs, no difference in distribution between males and females was observed. The mean age of the patients was 48.36 years (range, 13-77 years), as shown in Table 1. 
The three most common complaints were abdominal pain $(36.93 \%)$, bleeding manifestations either from the upper or lower gastrointestinal tract (16.66\%), and constipation (13.09\%). The remaining complaints were incidental findings of an abdominal mass, which were asymptomatic $(11.9 \%)$, symptoms related to metastatic location $(8.33 \%)$, such as fracture, functional tumor symptoms $(4.76 \%)$ with gastrinoma (indigestion, heartburn, and vomitus) and insulinoma (recurrent hypoglycemia), jaundice (7.14\%), and difficulty swallowing (1.19\%).

Table 1: Clinical characteristics of the samples $(n=84)$

\begin{tabular}{|c|c|}
\hline Variables & Frequency, percentage, mean, $S^{a}$ \\
\hline Age, mean (SD) & $48.36(16.69)$ years \\
\hline Tumor size, mean (SD) & $4.77(3.44) \mathrm{cm}$ \\
\hline \multicolumn{2}{|l|}{ Sex, n (\%) } \\
\hline Male & $42(50)$ \\
\hline Female & $42(50)$ \\
\hline \multicolumn{2}{|l|}{ Primary sites, n (\%) } \\
\hline Esophagus & $1(1.2)$ \\
\hline Stomach & $7(8.3)$ \\
\hline Small intestine & $7(8.4)$ \\
\hline Cecum & $2(2.4)$ \\
\hline Appendix & $4(4.8)$ \\
\hline Colon & $13(15.5)$ \\
\hline Rectum & $18(21.4)$ \\
\hline Pancreas & $15(17.9)$ \\
\hline Ampulla of Vater & $4(4.8)$ \\
\hline Unknown & $13(15.5)$ \\
\hline \multicolumn{2}{|l|}{ Staging, n (\%) } \\
\hline Localized & $45(53.6)$ \\
\hline Locoregional & $15(17.9)$ \\
\hline Disseminated & $24(28.6)$ \\
\hline \multicolumn{2}{|l|}{ Metastasis $(n=24), n(\%)$} \\
\hline Liver & $17(70.8)$ \\
\hline Renal & $2(8.3)$ \\
\hline Lung & $2(8.3)$ \\
\hline Bone & $1(4.2)$ \\
\hline Multiple organs & $2(8.3)$ \\
\hline \multicolumn{2}{|l|}{ Hormone status, n (\%) } \\
\hline Functioning & $8(9.5)$ \\
\hline Non-functioning & $76(90.5)$ \\
\hline
\end{tabular}

\section{Tumor characteristics}

The most common primary site was the rectum followed by the pancreas. Only eight patients had functioning tumors. Among them, seven cases were located in the pancreas and one in the duodenum. The average tumor size was $4.77 \mathrm{~cm}$. More than half of the tumors were localized, some were disseminated, and a few exhibited locoregional extension. Metastasis was predominantly found in the liver, followed by the lungs, kidneys, and bones. Two patients had multiple organ metastases both to the bone and liver.

\section{Histopathological characteristics}

The most common GEP-NET type was NET, followed by MiNEN, while the least common was NEC, as shown in Table 2. Of all the NETs, NET G2 was the most frequent. Of the NECs, SCNEC was the most frequent. LVI was present in 30 cases. The diagnosis of GEP-NET was established by positivity for chromogranin A or positivity for at least two of three neuroendocrine markers. The most common site with negative chromogranin A expression was the large intestine (eight cases; 36\%). Figure 1 shows microscopic images of GEP-NET from $\mathrm{HE}$ staining.

Table 2: Histopathological characteristics of the samples ( $\mathrm{n}=84)$

\begin{tabular}{ll}
\hline Variables & Frequency (percentage) \\
\hline Specimen type & \\
Biopsy & $46(54.8)$ \\
Resection & $38(45.2)$ \\
GEP-NET type & $32(38.1)^{\mathrm{a}}$ \\
NET & $11(34.4)^{\mathrm{b}}$ \\
NET G1 & $16(50)^{\mathrm{b}}$ \\
NET G2 & $5(15.6)^{\mathrm{b}}$ \\
NET G3 & $24(28.6)^{\mathrm{a}}$ \\
NEC & $14(58.3)^{\mathrm{c}}$ \\
SCNEC & $10(41.7)^{\mathrm{c}}$ \\
LCNEC & $28(33.3)^{\mathrm{a}}$ \\
MiNEN & \\
Grading ( $=81), \mathrm{n}(\%)$ & $44(54,3)$ \\
High-grade & $31(45.7)$ \\
Low-grade & \\
Lymphovascular invasion, $\mathrm{n}$ (\%) & $30(35.7)$ \\
Positive & $15(17.9)$ \\
Negative & $39(46.4)$ \\
Not available &
\end{tabular}

\section{Treatment and radiologic modalities}

Of the 84 patients, surgery was the most common treatment modality. However, some patients received a combination of surgery and chemotherapy or radiotherapy. Endoscopic therapy was used by eight patients with tumors originating from the duodenum and rectum. Surgery was performed in 68 patients with a variety of primary sites, the most common of which was the pancreas (15.47\%), rectum (14.29\%), and unknown (10.71\%). Chemotherapy was administered in 23 patients, among which 20 patients also underwent surgeryand onealso received radiotherapy. Radiotherapy was given to 11 patients, 10 of whom received palliative treatment. A somatostatin analog was prescribed for eight patients; of these, five had functioning tumors. The radiologic modality most commonly used in GEP-NET

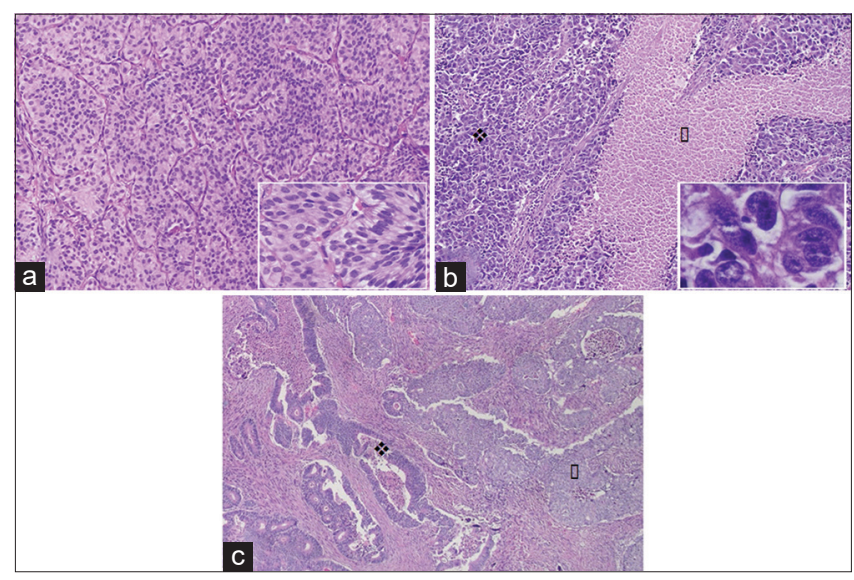

Figure 1: Microscopic finding of GEP-NETs from HE staining (a) Low-grade NET showing well-differentiated tumor (M100x, inlet: 400x); (b) high-grade NET showing poorly differentiated tumor (v) with a large area of necrosis ( $\star$ ) (M100x, inlet: 400x); (c) NET with adenocarcinoma component $(v)$ and neuroendocrine component ( $)(M 40 x)$ 
Table 3: Distribution of several diagnostic modalities in different primary sites of GEP-NETs

\begin{tabular}{|c|c|c|c|c|c|c|c|c|}
\hline \multirow[t]{2}{*}{ Primary sites } & \multicolumn{8}{|c|}{ Diagnosis modalities } \\
\hline & Upper endoscopy & Colonoscopy & Enteroscopy & Ultrasound & CT scan & MRI & ERCP/MRCP & Bone scan \\
\hline Esophagus & $1(100)$ & - & - & - & $1(100)$ & - & - & - \\
\hline Stomach & $6(85.7)$ & - & $1(14.3)$ & $3(42.9)$ & $6(85.7)$ & - & - & - \\
\hline Duodenum & $4(100)$ & - & - & $1(25)$ & $4(100)$ & - & - & - \\
\hline Jejunum & - & - & - & - & $1(100)$ & - & - & - \\
\hline Ileum & - & - & $2(100)$ & $2(100)$ & $2(100)$ & - & - & - \\
\hline Cecum & - & - & - & - & $2(100)$ & $1(50)$ & - & - \\
\hline Appendix & - & - & - & $4(100)$ & $3(75)$ & - & - & - \\
\hline Ascending colon & - & $7(100)$ & $1(14.3)$ & - & $6(85.7)$ & - & - & - \\
\hline Descending colon & - & $1(100)$ & - & - & $1(100)$ & - & - & - \\
\hline Sigmoid & - & $4(80)$ & - & - & $5(100)$ & - & - & - \\
\hline Rectum & - & $17(94.4)$ & - & - & $16(88.9)$ & - & - & - \\
\hline Ampulla of Vater & $1(25)$ & - & - & $2(50)$ & $4(100)$ & $1(25)$ & $4(100)$ & - \\
\hline Pancreas & $3(20)$ & - & - & $12(80)$ & $14(93.3)$ & $5(33.3)$ & $1(6.7)$ & - \\
\hline Unknown & - & - & - & $7(53.8)$ & $13(100)$ & $11(84.6)$ & - & $5(38.5)$ \\
\hline
\end{tabular}

cholangiopancreatography

patients was CT scan. Other modalities were dependent on the primary site, as shown in Table 3.

\section{Association between clinical and histopathological characteristics and tumor grade}

In all, 44 cases were high-grade tumors, and 37 were low-grade tumors. Of the 84 cases, three were excluded from the analysis because no Ki67 result was available. We performed a comparative analysis between tumor grade and age, primary site, gender, stage, LVI, tumor size, and metastasis. Tumor grade was significantly associated with the primary site, stage, LVI, tumor size, and metastasis. In contrast, age and sex were not significantly associated with tumor grade (Table 4). Post hoc analysis showed that lowgrade tumors tended to have a localized extension, while high-grade tumors tended to disseminate to other organs $(p=0.001)$. High-grade tumors had 6.5 times higher risk of LVI (OR 1.6-25). The mean size of highgrade tumors was $5.6 \mathrm{~cm}$, while the mean size of lowgrade tumors was $3.8 \mathrm{~cm}$, and this difference was statistically significant $(p=0.021)$. High-grade tumors had a 4.8 times greater risk of developing metastasis (OR 1.6-14.8). Post hoc analysis showed that tumors of an unknown primary site tended to be high grade, while those originating in the appendix tended to be low-grade tumors $(p=0.02)$.

\section{Discussion}

The number of GEP-NETs that we found in 10 years is low compared with what has been reported by Telli et al. who also performed a single-center study. However, there is a positive trend in the increment of cases each year, with the highest frequency in 2019 [7]. The limited knowledge of GEP-NETs among clinicians and pathologists in district hospitals may have contributed to the low number of GEP-NETs in our hospital, which, in these cases, was a referral hospital. In addition, most district hospitals are not yet equipped with immunohistochemistry laboratories. A study by Fan et al. obtained nationwide data on GEP-NETs
Table 4: Association between clinicopathologic characteristics and tumor grade $(n=81)$

\begin{tabular}{llll}
\hline Variables & High grade & Low grade & p-value \\
\hline Age, mean in years & 15.62 & 17.77 & $0.270^{\mathrm{a}}$ \\
Sex, $\mathrm{n}(\%)$ & & & \\
$\quad$ Male & $20(24.7)$ & $21(25.9)$ & $0.311^{\mathrm{b}}$ \\
$\quad$ Female & $24(29.6)$ & $16(19.8)$ & \\
Primary site, $\mathrm{n}(\%)$ & & & \\
$\quad$ Stomach & $5(6.2)$ & $2(2.5)$ & $0.020^{\mathrm{b}}$ \\
$\quad$ Small intestines & $5(6.2)$ & $2(2.5)$ & \\
$\quad$ Colorectal & $14(17.3)$ & $18(22.2)$ & \\
Pancreas & $7(8.6)$ & $8(9.9)$ & \\
$\quad$ Appendix & $0(0)$ & $4(4.9)$ & \\
$\quad$ Ampulla of Vater & $2(2.5)$ & $2(2.5)$ & \\
$\quad$ Unknown & $11(13.6)$ & $1(1.2)$ & \\
Staging, $\mathrm{n}(\%)$ & $16(19.8)$ & $27(33.3)$ & $0.003^{\mathrm{b}}$ \\
$\quad$ Localized & $9(11.1)$ & $5(6.2)$ & \\
$\quad$ Locoregional & $19(23.5)$ & $5(6.2)$ & \\
$\quad$ Disseminated & & & \\
Lymphovascular invasion, $\mathrm{n}(\%)$ & $23(28.4)$ & $7(8.6)$ & $0.006^{\mathrm{b}}$ \\
$\quad$ Positive & $5(6.2)$ & $10(12.3)$ & \\
$\quad$ Negative & 5.63 & 3.85 & $0.021^{\mathrm{b}}$ \\
Tumor size (cm) & & & \\
Metastasis, $\mathrm{n}(\%)$ & $19(23.5)$ & $5(6.2)$ & $0.004^{\mathrm{b}}$ \\
$\quad$ Yes & $25(30.9)$ & $32(39.5)$ & \\
$\quad$ No & Statistical analysis was performed by ${ }^{\mathrm{a}}$ independent sample t-test, and ${ }^{\mathrm{b}} \chi^{2}$ test. &
\end{tabular}

from 23 centers in China over 10 years. They found 2010 cases of GEP-NETs with an average of 89 cases per hospital [8]. This number is similar to that in our study, performed at a single national referral hospital.

This study shows that GEP-NET patients were not significantly different in terms of sex and that GEPNETs were most frequently found in patients older than 50 years. Three cases were pediatric GEP-NETs, while 38 were adults. Similar to our study, Lee et al. reported that older populations ( $\geq 50$ years of age) have a higher incidence of GEP-NET than younger individuals. However, they also found 37 cases of GEP-NET in children and teenagers [9]. We often find it challenging to diagnose GEP-NET in children since GEP-NET is not the first differential diagnosis in the round cell tumor group in the pediatric population.

The symptoms of GEP-NET are often nonspecific and are dependent on the primary site. Some are related to the mass effect and hormone status, but they are also dependent on the primary site. When the tumor originates from the esophagus, patients may complain of dysphagia. Moreover, small intestinal tumors can lead to jaundice and obstruction. Patients with colon tumors may present with bleeding manifestations and abdominal pain due to a tumor 
mass effect [2]. Our study showed that most patients presented to the hospital with a chief complaint of abdominal pain (either unspecified or colicky pain) followed by bleeding manifestations and constipation due to tumor obstruction.

The most frequent primary site in our study was the rectum, followed by the pancreas. Santos et al. reported a study from Portugal that showed that GEP. NET predominantly arises from the pancreas followed by the jejunum-ileum, stomach, and rectum [10]. Similar results were reported in a multicenter study by Cong et al. that the most common primary site was the pancreas followed by the rectum [11]. In addition, Fitzgerald et al. reported that the most common primary site was the small intestine followed by the colorectum and the pancreas; this is similar to what was published in the SEER program database, which reported that the five most common primary sites are the small intestine, rectum, pancreas, stomach, and appendix in that order [1], [12]. Our result was similar to those of the previous studies. Concerning to tumor grade, high-grade tumors were more frequent than low-grade tumors in our study. Of the NETs, G2 was the most frequent followed by $\mathrm{G} 1$ and $\mathrm{G} 3$. These results are different from other studies reported, which reported a higher frequency of low-grade tumors than high-grade tumors [7], [10], [12]. However, we are limited in sample size and study population, and thus, a nationwide epidemiology study might not be comparable to ours. A further large-scale epidemiology study of GEP-NETs in Indonesia should be performed.

Carbonero et al. conducted a GEP-NET study between 2001 and 2008. That study reported 837 cases: Disseminated tumors were the most frequent followed by localized tumors and those with locoregional extension. The liver was the most common metastatic site. The most common primary site from which the tumor disseminated to other organs was the small intestine, followed by the colon, rectum, and pancreas [6]. The previous study also reported that the most common tumor stage was disseminated, followed by localized and locoregional extension, with the liver as the most common metastatic site [10]. Most tumors in our study were localized. Some were disseminated, and very few exhibited locoregional extension. The most common metastatic site in our study was the liver. We found a significant association between the primary site and tumor expansion, where a tumor from an unknown primary site had the propensity to disseminate, and appendix-derived tumors tended to be localized. Identifying the primary site of an already disseminated tumor at the time of diagnosis was difficult, and immunohistochemistry staining, such as with PDX1 and CDX2 markers, is needed to confirm the origin of the tumor. However, these panels may provide overlapping results in determining the primary site. GEP-NETs of unknown primary account for $10-20 \%$ of all GEP-NETs and present most often as liver metastases, while the majority of metastases originate from the primary sites of the small intestine, pancreas, rectum, bile duct, and stomach [13]. GEP-NET of unknown primary comprises a relatively high number of tumors in our study. This might be due to a lack of available screening or a late diagnosis, which is why patients may have presented to the hospital with advanced stage disease. Furthermore, tests for specific markers to identify tumors of unknown origin were not available at our hospital.

In our study, the organ with the largest proportion of negative chromogranin $A$ expression was the large intestine. Chromogranin $A$, which can be negative, especially in the rectum, may become focally positive in G3 NETs and NECs [10]. Kimura et al. reported that $76 \%$ of NETs in the rectum were negative or focally positive for chromogranin A, but all cases of rectal NETs were positive for chromogranin B [14]. Fahrenkamp et al. also found that chromogranin B, but rarely chromogranin $A$, was strongly expressed in rectal NETs [15].

Carbonero et al. performed multivariate analysis and found that tumor expansion and $\mathrm{Ki} 67$ index were the only independent prognostic factors for survival [6]. Cho et al. reported that primary site, GEP-NET type, tumor extension, tumor size, mitosis, Ki67 index, LVI, perineural invasion, lymph node metastasis, and synaptophysin expression had prognostic significance [16]. Telli et al. reported that stage, grade, metastasis, and Ki67 index were significantly related to survival. Clinical Stage $3 / 4$, higher grade, metastasis, and higher Ki67 index were significant poor prognostic factors. In contrast, age, gender, and primary site were not significantly associated with survival [7]. Fitzgerald et al. performed a study from 2004 to 2013 with 39.454 patients with GEPNETs, of whom $84 \%$ had low-grade tumors. They also reported that grade had a significant relationship with age, tumor size, and primary site. High-grade tumors were more likely to be found in older patients and tended to have tumors larger than $4 \mathrm{~cm}$. Concerning the primary site, a large proportion of high-grade tumors originated in the esophagus, while the minor proportion originated from the small intestine [12]. We found that grade is significantly related to the primary site, stage, LVI, tumor size, and metastasis. Our study found that the mean size of high-grade tumors was $5.6 \mathrm{~cm}$, while the mean size of low-grade tumors was $3.8 \mathrm{~cm}$. Tumors from an unknown primary site have a 12 times greater risk of high-grade tumors than those from a specified primary site. We did not find any significant association between grade and age.

MiNENs are rare neoplasms reported to have a poor prognosis, and therefore, they are managed as non-neuroendocrine cancers. Colorectal MiNENs are among the most frequent types of MiNENs. De Mestier et al. proposed a classification system for MiNENs, whereby they are considered low, intermediate, or high grades. Low-grade MiNENs encompass adenomas and NET G1-2, intermediate MiNENs encompass 
carcinomas, and NET G1-2, while high-grade MiNENs consist of carcinomas or adenomas with NET G3 or NEC [17]. Frizziero et al. performed a retrospective study of MiNEN cases from five European centers. They reported 69 cases from 1980 to 2017, and the most common site of origin was the colorectal region, and of those, $73.4 \%$ were metastatic [18]. In all of our cases, MiNENs were predominantly high-grade tumors, followed by intermediate grades. The most common primary site was the large intestine followed by the pancreas. Of all the patients with MiNEN, 30.8\% presented with metastasis at the time of diagnosis.

\section{Conclusion}

Our study included 84 cases of GEP-NETs over 10 years; the mean patient age was 48.36 years. Most of these cases were high-grade, localized tumors originating from the rectum and pancreas, and most were non-functioning and were diagnosed as NETs. We also demonstrated that grade was significantly associated with the primary site, tumor expansion, LVI, and tumor size. An accurate pathology diagnosis, including an immunohistochemical analysis, is crucial in the management of GEP-NETs. Hence, increased awareness of GEP-NETs among clinicians and pathologists and the increased availability of diagnostic resources in district hospitals are essential. Further large-scale epidemiology studies of GEP-NET in Indonesia should be conducted to bring national attention to this disease entity.

\section{References}

1. Dasari A, Shen C, Halperin D, Zhao B, Zhou S, Xu Y, et al Trends in the incidence, prevalence, and survival outcomes in patients with neuroendocrine tumors in the United States. JAMA Oncol. 2017;3(10):1335-42. https://doi.org/10.1001/ jamaoncol.2017.0589

PMid:28448665

2. Nagtegaal ID, Odze RD, Klimstra D, Paradis V, Rugge M, Schirmacher P, et al. The 2019 WHO classification of tumours of the digestive system. Histopathology. 2020;76(2):182-8. https:// doi.org/10.1111/his.13975

PMid:31433515

3. Chan DT, Luk A, So WY, Kong A, Chow F, Ma R, et al. Natural history and outcome in chinese patients with gastroenteropancreatic neuroendocrine tumours: A 17-year retrospective analysis. BMC Endocr Disord. 2016;16:12. https:// doi.org/10.1186/s12902-016-0087-9

PMid: 26911576

4. Klöppel G, Rindi G,AnlaufM, PerrenA, Komminoth P. Site-specific biology and pathology of gastroenteropancreatic neuroendocrine tumors. Virchows Arch. 2007;451 Suppl 1:S9-27. https://doi. org/10.1007/s00428-007-0461-0

PMid:17684761

5. Oronsky B, Ma PC, Morgensztern D, Carter CA. Nothing but NET: A review of neuroendocrine tumors and carcinomas. Neoplasia. 2017;19(12):991-1002. https://doi.org/10.1016/j. neo.2017.09.002

PMid:29091800

6. Garcia-Carbonero R, Capdevila J, Crespo-Herrero G, DíazPérez JA, Del Prado MP, Orduña VA, et al. Incidence, patterns of care and prognostic factors for outcome of gastroenteropancreatic neuroendocrine tumors (GEP-NETs): Results from the national cancer registry of Spain (RGETNE). Ann Oncol. 2010;21(9):1794803. https://doi.org/10.1093/annonc/mdq022 PMid:20139156

7. Telli TA, Esin E, Yalçin Ş. Clinicopathologic features of gastroenteropancreatic neuroendocrine tumors: A singlecenter experience. Balkan Med J. 2020;37(5):281-6. https://doi. org/10.4274/balkanmedj.galenos.2020.2020.1.126 PMid:32573179

8. Fan JH, Zhang $Y Q$, Shi SS, Chen $Y J$, Yuan $X H$, Jiang LM, et al. A nation-wide retrospective epidemiological study of gastroenteropancreatic neuroendocrine neoplasms in china. Oncotarget. 2017;8(42):71699-708. https://doi.org/10.18632/ oncotarget.17599

PMid:29069739

9. Lee MR, Harris C, Baeg KJ, Aronson A, Wisnivesky JP Kim MK. Incidence trends of gastroenteropancreatic neuroendocrine tumors in the United States. Clin Gastroenterol Hepatol. 2019;17(11):2212-7.e1. https://doi.org/10.1016/j. cgh.2018.12.017 PMid:30580091

10. Santos AP, Vinagre J, Soares P, Claro I, Sanches AC, Gomes L, et al. Gastroenteropancreatic neuroendocrine neoplasia characterization in Portugal: Results from the nets study group of the portuguese society of endocrinology, diabetes and metabolism. Int J Endocrinol. 2019;2019:4518742. https://doi. org/10.1155/2019/4518742

PMid:31467527

11. Cong L, Wu W, Lou W, Wang J, Gu F, Qian J, et al. Gastroenteropancreatic neuroendocrine tumor registry study in China. J Pancreatol. 2018;1:35-8. https://doi.org/10.1097/ JP9.0000000000000005

12. Fitzgerald TL, Mosquera C, Lea CS, Mcmullen M. Primary site predicts grade for gastroenteropancreatic neuroendocrine tumors. Am Surg. 2017;83(7):799-803. https://doi. org/10.1177/000313481708300741 PMid:28738955

13. Chai SM, Brown IS, Kumarasinghe MP. Gastroenteropancreatic neuroendocrine neoplasms: Selected pathology review and molecular updates. Histopathology. 2018;72(1):153-67. https:// doi.org/10.1111/his.13367

PMid:29239038

14. Kimura N, Pilichowska M, Okamoto H, Kimura I, Aunis D Immunohistochemical expression of chromogranins $\mathrm{A}$ and $\mathrm{B}$, prohormone convertases 2 and 3 , and amidating enzyme in carcinoid tumors and pancreatic endocrine tumors. Mod Pathol. 2000;13:140-6. https://doi.org/10.1038/modpathol.3880026 PMid: 10697270

15. Fahrenkamp AG, Wibbeke C, Winde G, Ofner D, Böcker W, Fischer-Colbrie R, et al. Immunohistochemical distribution of chromogranins A and B and secretogranin II in neuroendocrine tumours of the gastrointestinal tract. Virchows Arch. 1995;426(4):361-7. https://doi.org/10.1007/BF00191345 PMid:7599788

16. Cho MY, Kim JM, Sohn JH, Kim MJ, Kim KM, Kim WH, et al 
Current trends of the incidence and pathological diagnosis of gastroenteropancreatic neuroendocrine tumors (GEPNETs) in Korea 2000-2009: Multicenter study. Cancer Res Treat. 2012;44(3):157-65. https://doi.org/10.4143/ crt.2012.44.3.157

PMid:23091441

17. de Mestier L, Cros J, Neuzillet C, Hentic O, Egal A, Muller N, et al Digestive system mixed neuroendocrine-non-neuroendocrine neoplasms. Neuroendocrinology. 2017;105(4):412-25. https:// doi.org/10.1159/000475527

PMid:28803232

18. Frizziero $M$, Wang $X$, Chakrabarty $B$, Childs A, Luong TV, Walter $\mathrm{T}$, et al. Retrospective study on mixed neuroendocrine non-neuroendocrine neoplasms from five European centres. World J Gastroenterol. 2019;25(39):5991-6005. https://doi. org/10.3748/wjg.v25.i39.5991

PMid:31660035 